The Astrophysical Journal, 648: L135-L138, 2006 September 10

(C) 2006. The American Astronomical Society. All rights reserved. Printed in U.S.A.

\title{
MID-INFRARED EMISSION FROM DUST AROUND QUIESCENT LOW-MASS X-RAY BINARIES
}

\author{
Michael P. Muno ${ }^{1,2}$ and Jon Mauerhan \\ Department of Physics and Astronomy, University of California, Los Angeles, 430 Portola Plaza, Box 951547, Los Angeles, CA 90095; \\ mmuno@astro.ucla.edu, mauerhan@astro.ucla.edu \\ Received 2006 May 17; accepted 2006 July 27; published 2006 August 24
}

\begin{abstract}
We report the discovery of excess 4.5 and $8 \mu \mathrm{m}$ emission from three quiescent black hole low-mass X-ray binaries, A0620-00, GS 2023+338, and XTE J1118+480, and the lack of similar excess emission from Cen X-4. The mid-infrared emission from GS $2023+338$ probably originates in the accretion disk. However, the excess emission from A0620-00 and XTE J1118+480 is brighter and peaks at longer wavelengths, and thus most likely originates from circumbinary dust that is heated by the light of the secondary star. For these two sources, we find that the inner edges of the dust distributions lie near 1.7 times the binary separations, which are the minimum radii at which circumbinary disks would be stable against tidal disruption. The excesses are weak at $24 \mu \mathrm{m}$, which implies that the dust does not extend beyond about 3 times the binary separations. The total masses of circumbinary material are between $10^{22}$ and $10^{24} \mathrm{~g}$. The material could be the remains of fallback disks produced in supernovae, or material from the companions injected into circumbinary orbits during mass transfer.
\end{abstract}

Subject headings: circumstellar matter -

stars: individual (A0620-00, Centaurus X-4, GS 2023+338, XTE J1118+480)

\section{INTRODUCTION}

One of the main limiting factors in determining the masses of the compact objects in low-mass X-ray binaries (LMXBs) is uncertainty in the fraction of the infrared light that is produced by the companion star (e.g., Hynes et al. 2005). When LMXBs are accreting at low rates, the optical and infrared light from these systems is dominated by the companion stars, so their mass functions can be measured to high accuracy based on the Doppler motion of the companion (e.g., McClintock \& Remillard 2006). The inclination then must be constrained by modeling the modulations in the optical and infrared light curves that are produced by the varying aspect of the distorted, Roche-lobe-filling companion. Generally, the contribution of the accretion disk to the optical and near-infrared emission is uncertain, and varying the fraction of the light that is assumed to be produced by the accretion disk can lead to differences of a factor of 2 in the derived mass of a compact object (e.g., Gelino et al. 2001). Fortunately, the broadband spectrum of a multitemperature accretion disk is significantly flatter than that of a stellar photosphere, so mid-infrared observations could constrain the relative contributions of the two components.

However, the spectra of LMXBs in the mid-infrared have not been well studied, and several indirect lines of evidence suggest that these systems might contain circumbinary material that could emit in the mid-infrared. First, some of the white dwarf analogs to LMXBs, cataclysmic variables (CVs), exhibit spectral features that lie at the mean radial velocities of the systems (Solheim \& Sion 1994) and excess mid-infrared emission (Howell et al. 2006) that could be interpreted as arising in circumbinary material (see also Taam et al. 2003; Dubus et al. 2004). Second, the supernovae that produced the compact objects could have left fallback disks around the binaries. Indeed, the first fallback disk has recently been found around a young, highly magnetized neutron star (Wang et al. 2006).

\footnotetext{
${ }^{1}$ Currrent address: Space Radiation Laboratory, California Institute of Technology, Pasadena, CA 91125; mmuno@ srl.caltech.edu.

${ }^{2}$ Hubble Fellow.
}

Third, the planets around the isolated millisecond pulsar PSR $1257+12$ (Wolszczan \& Frail 1992) could not have survived the supernova that produced the neutron star and must have formed afterward (see, e.g., Miller \& Hamilton 2001). Millisecond pulsars are usually assumed to have been spun up by accretion as LMXBs, in which case planets could form from material present during the binary phase. Therefore, to search for evidence of circumbinary material, we have observed four nearby, quiescent LMXBs with the Spitzer Space Telescope.

\section{OBSERVATIONS AND DATA ANALYSIS}

We chose the LMXBs in our sample to be detectable with Spitzer if they contained optically thick circumbinary disks passively illuminated by the mass donor stars (see Jura 2003; Taam et al. 2003 and below). Guided by a simple model, we chose sources with (1) $K$ magnitudes brighter than 17, (2) locations more than $50^{\circ}$ in projection from the Galactic center, and (3) no Two Micron All Sky Survey (2MASS) sources within $5^{\prime \prime}$ that were brighter than our targets. In Table 1 we list the positions, the orbital periods, estimates of the primary masses, the spectral types of the companions, and the quiescent $K$ magnitudes of the four systems in our sample (see the table notes for references).

Our measurements were taken in the 4.5 and $8.0 \mu \mathrm{m}$ bands with the Infrared Array Camera (IRAC), and in the $24 \mu \mathrm{m}$ band with the Multiband Imaging Photometer (MIPS; Table 2). We used the post-Basic Calibrated Data provided by the Spitzer Science Center (SSC) for most of our analysis. However, the MIPS image of A0620-00 contained latent features with a low spatial frequency and a $\sim 2 \%$ amplitude that were left by a previous observation of a bright, extended source. We corrected the image by creating a flat field from the median of the individual dithered images, dividing each snapshot by the flat, and recreating the mosaicked image using the script provided by the SSC. ${ }^{3}$ Threecolor images centered on each LMXB are displayed in Figure 1. Each target is detected at 4.5 and $8.0 \mu \mathrm{m}$. Only A0620-00 and GS $2023+338$ are also detected at $24 \mu \mathrm{m}$. We computed the IRAC

\footnotetext{
${ }^{3}$ See http://ssc.spitzer.caltech.edu/postbed.
} 
TABLE 1

TARgET QUIESCENT LMXBs

\begin{tabular}{|c|c|c|c|c|c|c|c|c|c|}
\hline Source & Optical & $\begin{array}{c}\text { R.A. } \\
(\mathrm{J} 2000.0)\end{array}$ & $\begin{array}{c}\text { Decl. } \\
(\mathrm{J} 2000.0)\end{array}$ & $\begin{array}{c}D \\
(\mathrm{kpc})\end{array}$ & $\begin{array}{l}P_{\text {orb }} \\
(\mathrm{hr})\end{array}$ & $\begin{array}{c}M_{1} \\
\left(M_{\odot}\right)\end{array}$ & Spectral Type & $A_{V}$ & $K$ \\
\hline A0620-00 $\ldots$ & V616 Mon & 95.68561 & -0.345628 & $1.2 \pm 0.1$ & 7.8 & $8.7-12.9$ & K4 V & 1.2 & $14.55(6)$ \\
\hline GS $2023+338 \ldots$ & V404 Cyg & 306.01594 & +33.86728 & $2.2-3.7$ & 155.3 & $10.1-13.4$ & K0 III & 4.0 & $12.50(5)$ \\
\hline XTE J1118+480 ...... & KV UMa & 169.54498 & +48.03678 & $1.8 \pm 0.5$ & 4.1 & $6.5-7.2$ & K6 V & 0.06 & $16.9(2)$ \\
\hline Cen $\mathrm{X}-4 \ldots \ldots \ldots \ldots$ & V822 Cen & 224.59135 & -31.66872 & 1.2 & 15.1 & $1.4^{\mathrm{a}}$ & $\mathrm{K} 5 \mathrm{~V}$ & 0.3 & $14.66(8)$ \\
\hline
\end{tabular}

Notes. - Units of right ascension are hours, minutes, and seconds, and units of declination are degrees, arcminutes, and arcseconds. The positions are taken from 2MASS, and the distances, orbital periods, and mass function are from McClintock \& Remillard (2006). For the remaining data, the references are as follows: (1) A0620-00: Gelino et al. (2001) for all photometry and the spectral type; (2) GS 2023+338: Casares et al. (1993) for all photometry and the spectral type; (3) XTE J1118+480: spectral type from Torres et al. (2004), extinction from McClintock et al. (2003), $J$ and $K$ from Mikołajewsa et al. (2005), $B$ from USNO, and $R$ from Zurita et al. (2002); (4) Cen X-4: spectral type from Shahbaz et al. (1993) and Torres et al. (2002), J, $H$, and $K$ from 2MASS; other bands from Shahbaz et al. (1993).

${ }^{a}$ We assumed this mass for the neutron star based on those measured for binary radio pulsars (Thorsett \& Chakrabarty 1999).

fluxes of each source using the point-spread function fitting routine APEX from the SSC, and the MIPS fluxes and upper limits using aperture photometry (Table 2 and Fig. 2).

To understand the origin of the mid-infrared emission, in Figure 2 we plot for each LMXB the observed and dereddened fluxes in the infrared and optical bands (from Table 2 and the references in Table 1). The optical and near-infrared intensities of quiescent LMXBs often vary by several tenths of a magnitude on timescales of years (not counting outbursts; Chevalier et al. 1989; Gelino et al. 2001), presumably because of changes in the accretion flow. None of the fluxes were obtained simultaneously, so when comparing the flux measurements in Figure 2 one can only expect them to be self-consistent to within $\approx 30 \%$. To estimate the contributions of the companions to the spectra, we have obtained model stellar spectra computed with the next-generation Phoenix code (kindly provided by $\mathrm{T}$. Barman; Hauschildt et al. 2001) that correspond to the temperatures and radii of the spectral types in Table 1 . We have estimated the contribution of the accretion disk using a standard Shakura \& Sunyaev (1973) model, assuming that the disk extends out to the tidal truncation radius ( $\$ 60 \%$ of the binary separation; Frank et al. 1992), is inclined by $60^{\circ}$ to our line of sight, and has the largest accretion rate that is consistent with the optical photometry (see the caption of Fig. 2 for values). We have confirmed that our conclusions are robust against changing the assumed spectral types of the companions within the values reported in the literature, and against varying the parameters of the accretion disk so long as the models are consistent with the optical photometry.

We find that the spectrum of Cen X-4 is consistent with that expected for the companion star, with a possible contribution at long wavelengths from the accretion disk. There are clear ex- cesses of mid-infrared flux above that expected from the companions of A0620-00, GS 2023+338, and XTE J1118+480.

In order to conservatively estimate the amounts of the midinfrared fluxes that are not produced by the companions, we computed the maximum contributions of the companions' photospheres by normalizing the model stellar spectra to match the dereddened $K$ fluxes, and we reported the difference between the predicted and observed mid-infrared fluxes as the excesses in Table $2\left(\Delta S_{\lambda}\right)$.

\section{DISCUSSION}

Several LMXBs have now been detected in outburst in the mid-infrared (e.g., Smith et al. 1990; van Paradijs et al. 1994; Harrison \& Gehrz 1991; Harrison \& Gehrz 1994; Migliari et al. 2006), but these are the first detections of quiescent systems. In outburst, the mid-infrared emission could originate from an accretion disk in which $\dot{M}>10^{18} \mathrm{~g} \mathrm{~s}^{-1}$ (Smith et al. 1990), from free-free emission in a strong wind driven from the accretion disk (van Paradijs et al. 1994), or from radio jets with flat spectra extending to infrared wavelengths (Fender 2001; Homan et al. 2005; Migliari et al. 2006). We use these hypotheses as a starting point for understanding the mid-infrared emission from our sample of quiescent LMXBs.

The mid-infrared excess from GS $2023+338$ could originate in an accretion disk, because the 2.2-24 $\mu \mathrm{m}$ fluxes in Figure 2 follow the $v^{-2}$ law that one would expect from the Rayleigh-Jeans tail of a blackbody. We find that our model for a viscously heated accretion disk (Shakura \& Sunyaev 1973) from $\S 2$ adequately describes the excess mid-infrared flux from GS $2023+338$ in Figure 2.

In contrast, the 4-24 $\mu \mathrm{m}$ fluxes from A0620-00 and XTE

TABLE 2

Spitzer Measurements of Quiescent LMXBs

\begin{tabular}{|c|c|c|c|c|c|c|c|c|c|c|}
\hline \multirow[b]{2}{*}{ SOURCE } & \multicolumn{6}{|c|}{ IRAC } & \multicolumn{4}{|c|}{ MIPS } \\
\hline & Date IRAC & $\begin{array}{c}T_{\text {IRAC }} \\
\text { (s) }\end{array}$ & $\begin{array}{c}S_{4.5} \\
(\mu \mathrm{Jy})\end{array}$ & $\begin{array}{l}\Delta S_{4.5} \\
(\mu \mathrm{Jy})\end{array}$ & $\begin{array}{c}S_{8.0} \\
(\mu \mathrm{Jy})\end{array}$ & $\begin{array}{l}\Delta S_{8.0} \\
(\mu \mathrm{Jy})\end{array}$ & Date MIPS & $\begin{array}{c}T_{\mathrm{MIPS}} \\
\text { (s) }\end{array}$ & $\begin{array}{c}S_{24} \\
(\mu \mathrm{Jy})\end{array}$ & $\begin{array}{c}\Delta S_{24} \\
(\mu \mathrm{Jy})\end{array}$ \\
\hline A0620-00 $\ldots \ldots$. & 2005 Mar 25 & 400 & $448(13)$ & 194 & $249(10)$ & 149 & 2005 Mar 06 & 180 & $54(18)$ & 43 \\
\hline GS $2023+338 \ldots \ldots \ldots$ & 2004 Oct 09 & 36 & $3020(90)$ & 670 & $1450(40)$ & 500 & 2004 Oct 16 & 30 & $153(70)$ & 46 \\
\hline XTE J1118+480 ..... & 2004 Nov 21 & 400 & $46(1)$ & 17 & $45(7)$ & 34 & 2005 May 13 & 240 & $<16$ & $<16$ \\
\hline Cen $\mathrm{X}-4 \ldots \ldots \ldots \ldots$ & 2004 Aug 12 & 300 & 199(6) & -90 & $95(17)$ & -14 & 2005 Aug 28 & 150 & $<30$ & $<30$ \\
\hline
\end{tabular}

Notes. - We list the date and duration of each observation (the 4.5 and $8 \mu \mathrm{m}$ data were obtained in parallel), the fluxes measured ( $S_{\lambda}$, where $\lambda$ is the central wavelength of each band), and estimates of the minimum amount of excess mid-infrared emission above that expected from the photospheres of the companions $\left(\Delta S_{\lambda}\right.$; see text). The quoted uncertainties are the larger of the standard deviation of the fluxes in the multiple frames we obtained, or the systematic uncertainty in the absolute calibration (3\% for IRAC and 5\% for MIPS). The negative values for Cen X-4 reflect the fact that extrapolating a $\lambda^{2}$ spectrum expected for the Rayleigh-Jeans tail of the photosphere from the $K$-band flux predicts more mid-infrared flux than is observed; this is likely because the infrared flux is variable. 


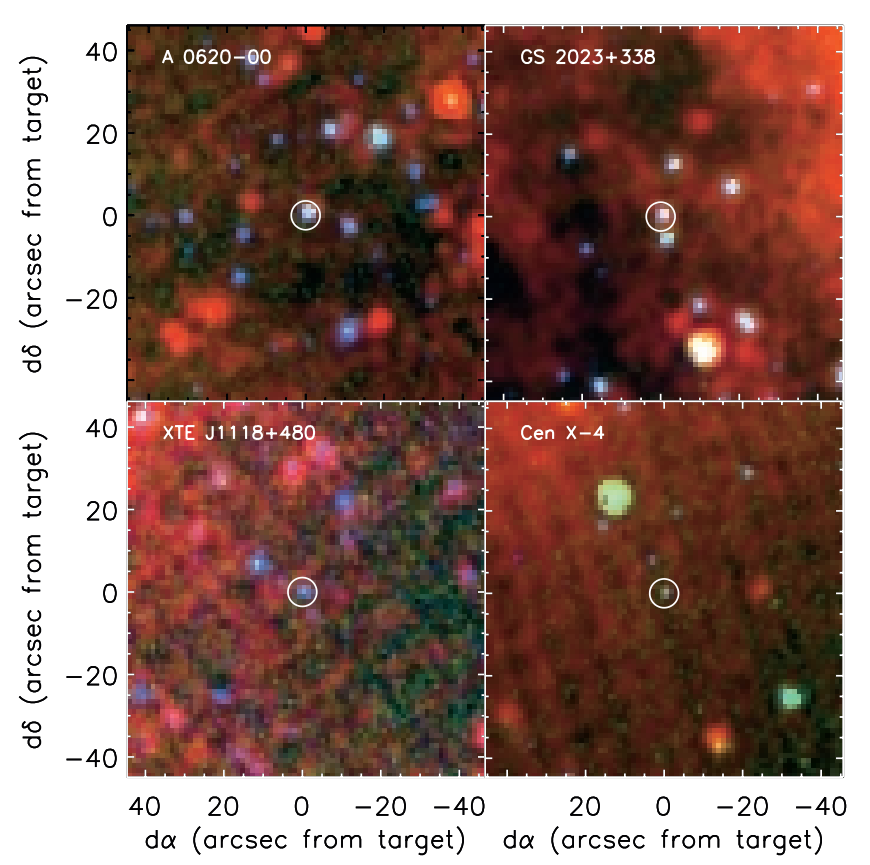

FIG. 1.-Mid-infrared images of the LMXBs in this survey. The red channel is $24 \mu \mathrm{m}$, the green is $8.0 \mu \mathrm{m}$, and the blue is $4.5 \mu \mathrm{m}$. The circles denote the positions of the optical counterparts. XTE J1118+480 and Cen X-4 were not detected at $24 \mu \mathrm{m}$.

$\mathrm{J} 1118+480$ do not follow the $\nu^{-2}$ law expected for the Rayleigh-Jeans tail of a blackbody, because there is too much flux at $8 \mu \mathrm{m}$. A blackbody spectrum that peaks near $8 \mu \mathrm{m}$ would have a temperature of only $640 \mathrm{~K}$. For these two sources, we plot blackbody spectra that match the peak of the mid-infrared excesses $\left(\Delta S_{8.0}\right)$ in Figure 2. The sum of the spectra of stellar photospheres and optically thick blackbodies match the observed mid-infrared fluxes well. However, the inferred emitting areas are large compared to the binary separations. For a Planck spectrum, the solid angles of the emitting regions correspond to circular radii of

$$
R=4 \times 10^{11}\left(\frac{\Delta S_{8 \mu \mathrm{m}}}{100 \mu \mathrm{Jy}}\right)^{1 / 2}\left(\frac{D}{\mathrm{kpc}}\right) \mathrm{cm}
$$

where $D$ is the distance to the source. In contrast, the disks will be contained within $\$ 60 \%$ of the binary separations $a$, which are given by

$$
a=3 \times 10^{11} M^{1 / 3}(1+q)^{1 / 3} P_{\mathrm{day}}^{2 / 3} \mathrm{~cm}
$$

where $P_{\text {day }}$ is the orbital period in days, $M$ is the mass of the accretor in solar masses, and $q$ is the mass ratio of the binary (e.g., Frank et al. 1992). Given the fluxes in Table 2, we find that the excess mid-infrared emission originates from regions $\approx 2$ times larger than the orbital separations of A0620-00 and $\mathrm{XTE} \mathrm{J} 1118+480$, and $\gtrsim 4$ times larger than the accretion disks. Therefore, the mid-infrared emission is produced in a region that extends beyond the binary orbit.

Jets could produce an emitting region larger than the binary orbit, but given the spectra of the mid-infrared excesses, we suggest that jets are only minor contributors. Two LMXBs in our sample have recently been detected as radio sources in quiescence: GS $2023+338$ with flat-spectrum radio emission with an intensity of $350 \mu \mathrm{Jy}$ over the frequency range $1.4-8.4 \mathrm{GHz}$ (Gallo et al. 2005),
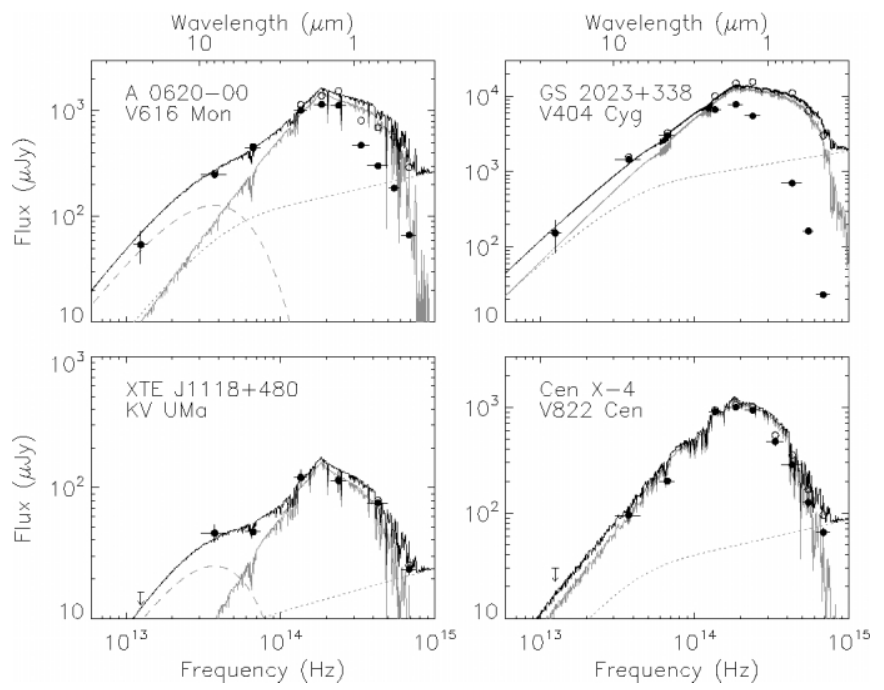

FIG. 2.-Spectra of the LMXBs in our sample. The filled circles are the observed fluxes in each band, and the open circles are the dereddened fluxes (see notes to Table 1 for references). The upper limits on the $24 \mathrm{~m}$ fluxes for XTE J1118+480 and Cen X-4 are indicated with arrows. The uncertainties in the observed fluxes are also indicated but are generally smaller than the symbol sizes. The solid gray lines are synthetic stellar spectra for the spectral type in Table 1 . The dotted gray lines are model spectra of viscously heated accretion disks, with accretion rates of $3 \times 10^{14} \mathrm{~g} \mathrm{~s}^{-1}$ for A0620-00, $7 \times$ $10^{16} \mathrm{~g} \mathrm{~s}^{-1}$ for GS $2023+338,4 \times 10^{13} \mathrm{~g} \mathrm{~s}^{-1}$ for XTE J1118+480, and $4 \times$ $10^{14} \mathrm{~g} \mathrm{~s}^{-1}$ for Cen X-4. The gray dashed lines illustrate the spectra of blackbodies used in the text to estimate the solid angles of the material responsible for the mid-infrared excesses from A0620-00 and XTE J1118+480. The black lines are the sum of these model components, normalized to match the dereddened $K$ flux.

and A0620-00 with a radio flux of $50 \mu \mathrm{Jy}$ at $8.4 \mathrm{GHz}$ (Gallo et al. 2006). The $24 \mu \mathrm{m}$ excesses from A0620-00 is equal to the flux in the radio, so it could be produced by a jet with a flat $\left(S_{\nu} \propto \nu^{\alpha}\right.$, with $\alpha \approx 0$ ) spectrum between the radio and mid-infrared. For GS $2023+338$, the $24 \mu \mathrm{m}$ flux is slightly lower than the radio flux, which would imply that its jet has a steeper $\alpha \gtrsim$ -0.3 spectrum. However, the $8 \mu \mathrm{m}$ excesses from A0620-00, GS $2023+338$, and XTE J1118+480 are not consistent with a flat-spectrum radio jet, because for XTE J1118+480 and A0262-00 they are 4 times larger than the excesses at $24 \mu \mathrm{m}$, and for GS $2023+338$ it is 10 times larger. If we assume the $24 \mu \mathrm{m}$ emission is from a flat-spectrum jet, then it contributes $<25 \%$ to the $8 \mu \mathrm{m}$ flux from XTE J1118+480 and A0620-00, and $<10 \%$ from GS $2023+338$.

We suggest that the excesses from A0620-00 and XTE J1118+ 480 originate from circumbinary dust that reprocesses the light of the companions. To estimate the masses of the dust, we assume it is contained in optically thick disks. The disks could lie as close to the center of masses of the binaries as $1.7 a$, at which point they would be tidally truncated (Taam et al. 2003; Dubus et al. 2004). We can estimate the temperature profile of such disks by assuming they are flat, in which case

$$
T_{\mathrm{disk}} \approx\left(\frac{2}{3 \pi}\right)^{1 / 4}\left(\frac{R_{*}}{R_{\mathrm{disk}}}\right)^{3 / 4} T_{*}
$$

where $T_{\text {disk }}$ and $R_{\text {disk }}$ are the temperatures and radii of the disks, and $T_{*}$ and $R_{*}$ are the temperatures and radii of the companions (e.g., Ruden \& Pollack 1991; Jura 2003). Using equation (2) and the parameters in Table 1, we would expect the inner edges of the circumbinary disks to have temperatures of $\approx 600 \mathrm{~K}$, which is consistent with our detection of excesses that peak 
near $8 \mu \mathrm{m}$. The lack of excess flux at $24 \mu \mathrm{m}$ implies that material does not reprocess much stellar light beyond radii $\approx 3$ times larger than the binary separations. If we assume the disks are composed of dust with an opacity $\chi_{\nu} \sim 300 \mathrm{~cm}^{2} \mathrm{~g}^{-1}$ at $8 \mu \mathrm{m}$ (e.g., Draine \& Lee 1984), then for them to have an optical depth of $\tau \sim 1$, the disks would only need to contain $\sim 10^{22} \mathrm{~g}$ of dust. If we assume the emitting material is optically thin, we find dust masses that are similar within a factor of a few (e.g., eq. [3] in Evans et al. 1997). If the gas-to-dust ratio is similar to that of the interstellar medium, $\sim 100$, then the total mass of the circumbinary material could be $\sim 10^{24} \mathrm{~g}$.

\section{CONCLUSIONS}

The detection of excess mid-infrared flux from A0620-00 and XTE J1118+480 (Fig. 2) provides evidence that circumbinary material is present around some LMXBs, but its spectrum suggests that it will only be detectable in the mid-infrared. The excess emission peaks at $8 \mu \mathrm{m}$, and if we model it as a single-temperature blackbody, we predict that the circumbinary material produces negligible flux shortward of $\approx 3 \mu \mathrm{m}$. Moreover, the lack of excess mid-infrared emission from Cen X-4 demonstrates that such material is not ubiquitous. Finally, for GS $2023+338$, the mid-infrared emission appears to originate from a hot accretion disk, and the contribution of the accretion disk to the mid-infrared light can be predicted based on the optical measurements (Fig. 2, dotted line). These results provide reassurance that efforts to determine the inclinations of LMXBs by modeling the ellipsoidal modulations of their infrared and optical light curves are not compromised by the presence of circumbinary material.

For A0620-00 and XTE J1118+480, we suggest that the circumbinary disks are either the remains of fallback disks produced in the supernovae that formed the compact objects (e.g., Wang et al. 2006; Cordes \& Shannon 2006), or material injected into circumbinary orbits during the process of mass loss by the Roche lobe-filling companions (e.g., Dubus et al. 2004). These circumbinary disks contain $\sim 10^{22} \mathrm{~g}$ of dust, which for a standard dust-to-mass ratio of $\sim 100$ implies a total mass of $\sim 10^{24} \mathrm{~g}$. If the disks are produced by matter ejected into circumbinary orbits during the process of mass transfer, then it represents only a tiny fraction of the $\sim 10^{32} \mathrm{~g}\left(\sim 0.1 \mathrm{M}_{\odot}\right)$ that will be lost by the companions over the lifetimes of these LMXBs. Alternatively, Cordes \& Shannon (2006) have proposed that fallback disks containing $\gtrsim 10^{24} \mathrm{~g}$ of material could form asteroids and inject them into the magnetospheres of pulsars with a high enough rate to explain the observed intermittency in their radio pulses. Asteroids might not survive the outbursts of LMXBs (Miller \& Hamilton 2001), but the midinfrared excesses that we have identified could be the remnants of similar fallback disks.

However, the circumbinary material is not massive enough either to affect the evolution of the orbital angular momentum of LMXBs (e.g., $\sim 10^{29} \mathrm{~g}$ in Taam et al. 2003), or to form planetesimals (e.g., $\sim 10^{28} \mathrm{~g}$ in Miller \& Hamilton 2001). As the formation and evolution of circumbinary matter around LMXBs and CVs are considered further, it may be that the paucity of matter around A0620-00 and XTE J1118+480 (and the apparent lack of circumbinary matter around the one neutron star LMXB in our sample, Cen X-4) may be the most lasting aspect of this result.

We are grateful to T. Barman for providing the model stellar spectra, to E. Gallo and S. Migliari for alerting us to a mistake in our original photometry, to M. Jura for conversations about circumstellar dust, to J. McClintock for discussions about the contribution of the accretion disk to the mid-infrared light, and to the referee for comments that clarified the text. Support for this work was provided by NASA through an award issued by JPL/Caltech.

\section{REFERENCES}

Casares, J., Charles, P. A., Naylor, T., \& Pavlenko, E. P. 1993, MNRAS, 265, 834

Chevalier, C., Ilovaisky, S. A., van Paradijs, J., Pedersen, J., \& van der Klis, M. 1989, A\&A, 210, 114

Cordes, J. M., \& Shannon, R. M. 2006, preprint (astro-ph/0605145)

Draine, B. T., \& Lee, H. M. 1984, ApJ, 285, 89

Dubus, G., Campbell, R., Kern, B., Taam, R. E., \& Spruit, H. C. 2004, MNRAS, 349, 869

Evans, A., Geballe, T. R., Rawlings, J. M. C., Eyres, S. P. S., \& Davies, J. K. 1997, MNRAS, 292, 192

Fender, R. P. 2001, MNRAS, 322, 31

Frank, J., King, A., \& Raine, D. 1992, Accretion Power in Astrophysics (Cambridge: Cambridge Univ. Press)

Gallo, E., Fender, R. P., \& Hynes, R. I. 2005, MNRAS, 356, 1017

Gallo, E., Fender, R. P., Miller-Jones, J. C. A., Merloni, A., Jonker, P. G.,

Heinz, S., Maccarone, T. J., \& van der Klis, M. 2006, MNRAS, 370, 1351

Gelino, D. M., Harrison, T. E., \& Orosz, J. A. 2001, AJ, 122, 2668

Harrison, T. E., \& Gehrz, R. D. 1991, AJ, 101, 587 1994, AJ, 108, 1899

Hauschildt, P. H., Lowenthal, D. K., \& Baron, E. 2001, ApJS, 134, 323

Homan, J., Buxton, M., Markoff, S., Bailyn, C. D., Nespoli, E., \& Belloni, T. 2005, ApJ, 624, 295

Howell, S. B., et al. 2006, ApJ, in press (astro-ph/0606202)

Hynes, R. I., Robinson, E. L., \& Bitner, M. 2005, ApJ, 630, 405

Jura, M. 2003, ApJ, 584, L91

McClintock, J. E., Narayan, R., Garcia, M. R., Orosz, J. A., Remillard, R. A., \& Murray, S. S. 2003, ApJ, 593, 435
McClintock, J. E., \& Remillard, R. A. 2006, in Compact Stellar X-ray Sources, ed. W. H. G. Lewin \& M. van der Klis (Cambridge: Cambridge Univ. Press), in press (astro-ph/0306213)

Migliari, S., Tomsick, J. A., Maccarone, T. J., Gallo, E., Fender, R. P., Nelemands, G., \& Russell, D. M. 2006, ApJ, 643, L41

Mikołajewsa, K., Ruthowski, A., Gonçalves, D. R., \& Szostek, A. 2005, MNRAS, 362, L13

Miller, M. C., \& Hamilton, D. P. 2001, ApJ, 550, 863

Ruden, S. P., \& Pollack, J. B. 1991, ApJ, 375, 740

Shahbaz, T., Naylor, T., \& Charles, P. A. 1993, MNRAS, 265, 655

Shakura, N. I., \& Sunyaev, R. A. 1973, A\&A, 24, 337

Smith, H. A., Beall, J. H., \& Swain, M. R. 1990, AJ, 99, 273

Solheim, S.-E., \& Sion, E. M. 1994, A\&A, 287, 503

Taam, R. E., Sandquist, E. L., \& Dubus, G. 2003, ApJ, 592, 1124

Thorsett, S. E., \& Chakrabarty, D. 1999, ApJ, 512, 288

Torres, M. A. P., Callanan, P. J., Garcia, M. R., Zhao, P., Laycock, S., \& Kong, A. K. H. 2004, ApJ, 612, 1026

Torres, M. A. P., Casares, J., Martínez-Pais, I. G., \& Charles, P. A. 2002, MNRAS, 334, 233

van Paradijs, J., Telesco, C. M., Kouveliotou, C., \& Fishman, G. J. 1994, ApJ, 429, L19

Wang, Z., Chakrabarty, D., \& Kaplan, D. L. 2006, Nature, 440, 772

Wolszczan, A., \& Frail, D. A. 1992, Nature, 355, 145

Zurita, C., Casares, J, Martinez-Pais, I. G., Piccioni, A., Bernabei, S., Bartolini, C., \& Guarnieri, A. 2002, IAU Circ. 7868 